\title{
Exponential Inequalities for Positive Linear Mappings
}

\author{
Mohammad Sababheh, ${ }^{1}$ Hamid Reza Moradi, ${ }^{2}$ and Shigeru Furuichi ${ }^{3}{ }^{3}$ \\ ${ }^{1}$ Department of Basic Sciences, Princess Sumaya University for Technology, Amman, Jordan \\ ${ }^{2}$ Young Researchers and Elite Club, Mashhad Branch, Islamic Azad University, Mashhad, Iran \\ ${ }^{3}$ Department of Computer Science and System Analysis, College of Humanities and Sciences, Nihon University, \\ 3-25-40 Sakurajyousui, Setagaya-ku, Tokyo 156-8550, Japan
}

Correspondence should be addressed to Shigeru Furuichi; furuichi@chs.nihon-u.ac.jp

Received 16 April 2018; Accepted 16 September 2018; Published 1 October 2018

Academic Editor: Aurelian Gheondea

Copyright (c) 2018 Mohammad Sababheh et al. This is an open access article distributed under the Creative Commons Attribution License, which permits unrestricted use, distribution, and reproduction in any medium, provided the original work is properly cited.

In this article, we present exponential-type inequalities for positive linear mappings and Hilbert space operators, by means of convexity and the Mond-Pečarić method. The obtained results refine and generalize some known results. As an application, we present extensions for operator-like geometric and harmonic means inequalities.

\section{Introduction}

Let $\mathscr{B}(\mathscr{H})$ be the $C^{*}$ algebra of bounded linear operators on a complex Hilbert space $\mathscr{H}$. If $A \in \mathscr{B}(\mathscr{H})$ is positive, we write $A \geq 0$. Further, we use the notation $\mathscr{B}^{+}(\mathscr{H})$ for the cone of all positive operators in $\mathscr{B}(\mathscr{H})$. For two self-ajoint operators $A, B \in \mathscr{B}(\mathscr{H})$, we write $A \leq B$ if $B-A \geq 0$. For a realvalued function $f$ of a real variable and a self-adjoint operator $A \in \mathscr{B}(\mathscr{H})$, the value $f(A)$ is understood by means of the functional calculus.

Let $J$ be a real interval of any type. A continuous function $f: J \longrightarrow \mathbb{R}$ is said to be operator convex if $f((1-v) A+$ $v B) \leq(1-v) f(A)+v f(B)$ holds for each $v \in[0,1]$ and every pair of self-adjoint operators $A, B \in \mathscr{B}(\mathscr{H})$, with spectra in $J$. The notation $\mathscr{B}_{[m, M]}^{+}(\mathscr{H})$ will be used in the sequel to denote the class of all positive operators $A \in \mathscr{B}(\mathscr{H})$ satisfying $m I \leq$ $A \leq M I$, for some positive scalars $m$ and $M$. A linear map $\varphi$ : $\mathscr{B}(\mathscr{H}) \longrightarrow \mathscr{B}(\mathscr{K})$ is said to be positive if $\varphi(A) \geq 0$ whenever $A \geq 0$. If, in addition, $\varphi(I)=I$, it is said to be normalized.

If $f: J \longrightarrow \mathbb{R}$ is operator convex, then, for any normalized positive linear map $\varphi$, we have $[1,2]$

$$
f(\varphi(A)) \leq \varphi(f(A))
$$

while we have the reversed inequality if $f$ is operator concave, for any self-adjoint operator $A$ with spectrum in $J$.
Inequality (1) is not true if $f$ is a convex function (rather than operator convex). However, in the interesting paper [3], various complementary inequalities have been presented for convex and concave functions. For example, it is shown that, for any positive number $\alpha$, one can find a constant $\beta$ such that

$$
\varphi(f(A)) \leq \alpha f(\varphi(A))+\beta,
$$

for the twice differentiable convex function $f:[m, M] \longrightarrow$ $\mathbb{R}$ and any self-adjoint operator $A$ on $\mathscr{H}$ with spectrum in $[m, M]$.

Earlier, it has been shown that, for any continuous real valued function $f$, one can find positive constants $\alpha$ and $\beta$ such that [4]

$$
\alpha \varphi(f(A)) \leq f(\varphi(A)) \leq \beta \varphi(f(A)) .
$$

In Proposition 10, we present a special case of (2) for a particular choice of $\alpha$; however we present a simple proof for completeness. Then as an application, we present several improvements and extensions of (2) and (3) for a log-convex function $f$.

In the sequel, we adopt the following notations. For a given function $f:[m, M] \longrightarrow \mathbb{R}$, define

$$
L[m, M, f](t)=a[m, M, f] t+b[m, M, f],
$$


where

$$
\begin{aligned}
a[m, M, f] & =\frac{f(M)-f(m)}{M-m} \\
\text { and } b[m, M, f] & =\frac{M f(m)-m f(M)}{M-m} .
\end{aligned}
$$

If no confusion arises, we will simply write $a[m, M, f]=a_{f}$ and $b[m, M, f]=b_{f}$.

Also, for $t_{0} \in(m, M)$, define

$$
L^{\prime}\left[t_{0}, f\right](t)=f\left(t_{0}\right)+f^{\prime}\left(t_{0}\right)\left(t-t_{0}\right),
$$

provided that $f^{\prime}\left(t_{0}\right)$ exists.

It is clear that, for a convex function $f:[m, M] \longrightarrow \mathbb{R}$, one has

$$
L^{\prime}\left[t_{0}, f\right](t) \leq f(t) \leq L[m, M, f](t),
$$

while the inequalities are reversed for a concave function $f$.

Remark 1. Notice that if $f$ is convex on an interval $J$ containing $[m, M]$, then (7) is still valid for any $t_{0} \in J$. That is, $t_{0}$ does not need to be in $(m, M)$.

Now, if $f:[m, M] \longrightarrow \mathbb{R}^{+}$is log-convex, we have the inequality $\log f(t) \leq L[m, M, \log f](t)$, which simply reads as follows

$$
\begin{aligned}
f(t) & \leq\left(f^{t-m}(M) f^{M-t}(m)\right)^{1 /(M-m)} \\
& \leq L[m, M, f](t), \quad m \leq t \leq M,
\end{aligned}
$$

where the second inequality is due to the arithmeticgeometric inequality. We refer the reader to [5] for some detailed discussion of (8).

Another useful observation about log-convex functions is the following. If $f$ is log-convex on $[m, M]$ and if $t_{0} \in(m, M)$ is such that $f\left(t_{0}\right) \neq 0$, (7) implies

$$
L^{\prime}\left[t_{0}, g\right](t) \leq g, \quad \text { where } g=\log f .
$$

Simplifying this inequality implies the following.

Lemma 2. Let $f:[m, M] \longrightarrow \mathbb{R}^{+}$be log-convex. If $f$ is differentiable at $t_{0} \in(m, M)$, then

$$
f(t) \geq f\left(t_{0}\right) \exp \left[\frac{f^{\prime}\left(t_{0}\right)}{f\left(t_{0}\right)}\left(t-t_{0}\right)\right], \quad m \leq t \leq M .
$$

In this article, we present several inequalities for logconvex functions based on the Mond-Pečarić method. In particular, we present inequalities that can be viewed as exponential inequalities for log-convex functions. More precisely, we present inequalities among the quantities

$$
\varphi(f(A)), f(\varphi(A)), \varphi\left(\left(f^{A-m}(M) f^{M-A}(m)\right)^{1 /(M-m)}\right)
$$

and

$$
\left(f^{\varphi(A)-m}(M) f^{M-\varphi(A)}(m)\right)^{1 /(M-m)} .
$$

Another interest in this paper is to present inequalities for operator-like means when filtered through normalized positive linear maps. That is, it is known that, for an operator mean $\sigma$ and two positive invertible operators $A, B$, one has [6]

$$
\varphi(A \sigma B) \leq \varphi(A) \sigma \varphi(B) .
$$

In particular, we show complementary inequalities for the geometric $\sharp_{t}$ and harmonic ! operator-like means, when $t<$ 0 . Of course, when $t<0$, these are not operator means. Our results can be considered as extensions of [7, Theorem 2.2].

\section{Main Results}

Now we proceed to the main results, starting with a complementary result of [8, Corollary 2.5] and [5, Proposition 2.1].

Proposition 3. Let $f:[m, M] \longrightarrow \mathbb{R}^{+}$be log-convex, $A \in$ $\mathscr{B}_{[m, M]}^{+}(\mathscr{H})$, and $\varphi$ be a normalized positive linear map. Then

$$
\begin{aligned}
& \frac{1}{\mu(m, M, f)} \varphi(f(A)) \\
& \quad \leq \frac{1}{\mu(m, M, f)} \varphi\left(\left(f^{A-m}(M) f^{M-A}(m)\right)^{1 /(M-m)}\right) \\
& \quad \leq f(\varphi(A)) \leq\left(f^{\varphi(A)-m}(M) f^{M-\varphi(A)}(m)\right)^{1 /(M-m)} \\
& \quad \leq \mu(m, M, f) \varphi(f(A))
\end{aligned}
$$

where

$$
\begin{aligned}
& \mu(m, M, f) \\
& \quad \equiv \max \left\{\frac{1}{f(t)}\left(\frac{M-t}{M-m} f(m)+\frac{t-m}{M-m} f(M)\right): m\right. \\
& \quad \leq t \leq M\} .
\end{aligned}
$$

Proof. The first and the second inequalities follow from [5, Proposition 2.1] and the fact that $\mu(m, M, f)>0$. So we have to prove the other inequalities. Applying a standard functional calculus argument for the operator $\varphi(A)$ in (8), we get

$$
\begin{aligned}
f(\varphi(A)) & \leq\left(f^{\varphi(A)-m}(M) f^{M-\varphi(A)}(m)\right)^{1 /(M-m)} \\
& \leq L[m, M, f](\varphi(A)) .
\end{aligned}
$$

Following [8], we have, for $\alpha>0$,

$$
\begin{aligned}
& L[m, M, f](\varphi(A))-\alpha \varphi(f(A)) \\
& \quad=a_{f} \varphi(A)+b_{f}-\alpha \varphi(f(A)) \leq \beta,
\end{aligned}
$$

where $\beta=\max _{t \in[m, M]}\left\{a_{f} t+b_{f}-\alpha f(t)\right\}$. That is,

$$
L[m, M, f](\varphi(A)) \leq \alpha \varphi(f(A))+\beta .
$$

By setting $\beta=0$, we obtain $\alpha=\max _{t \in[m, M]}\left\{\left(a_{f} t+\right.\right.$ $\left.\left.b_{f}\right) / f(t)\right\}:=\mu(m, M, f)$. With this choice of $\alpha$, we have $L[m, M, f](\varphi(A)) \leq \mu(m, M, f) \varphi(f(A))$, which, together with (16), complete the proof. 
Notice that Proposition 3 can be regarded as an operator extension of [9, Theorem 2.5] and a refinement of [8, Corollary 2.5].

Corollary 4. Let $A \in \mathscr{B}_{[m, M]}^{+}(\mathscr{H})$ and $\varphi$ be a normalized positive linear map. Then, for $t<0$,

$$
\begin{aligned}
& \frac{1}{K(m, M, t)} \varphi\left(A^{t}\right) \\
& \quad \leq \frac{1}{K(m, M, t)} \varphi\left(\left(M^{A-m} m^{M-A}\right)^{t /(M-m)}\right) \leq \varphi(A)^{t} \\
& \quad \leq\left(M^{\varphi(A)-m} m^{M-\varphi(A)}\right)^{t /(M-m)} \leq K(m, M, t) \varphi\left(A^{t}\right)
\end{aligned}
$$

where the generalized Kantrovich constant is defined by

$$
\begin{aligned}
& K(m, M, t) \\
& \quad=\frac{\left(m M^{t}-M m^{t}\right)}{(t-1)(M-m)}\left(\frac{t-1}{t} \frac{M^{t}-m^{t}}{m M^{t}-M m^{t}}\right)^{t} .
\end{aligned}
$$

Proof. The result follows immediately from Proposition 3, by letting $f(x)=x^{t}$.

Remark 5. Corollary 4 presents a refinement of the corresponding result in [10, Lemma 2].

As another application of Proposition 3, we have the following bounds for operator means. To simplify our statement, we will adopt the following notations. For a given function $f:[m, M] \longrightarrow[0, \infty)$ and two positive operators $A$ and $B$ satisfying $m A \leq B \leq M A$, we write

$$
\begin{aligned}
& A \sigma_{f} B:=A^{1 / 2} f\left(A^{-1 / 2} B A^{-1 / 2}\right) A^{-1 / 2} \\
& \text { and } A \delta B=A^{-1 / 2} B A^{-1 / 2} \text {. }
\end{aligned}
$$

Corollary 6. Let $A, B \in \mathscr{B}^{+}(\mathscr{H})$ be such that $m A \leq B \leq$ $M A$ for some positive scalars $m, M$. Then, for any linear map $\varphi$ (not necessarily normalized) and any log-convex function $f$ : $[m, M] \longrightarrow \mathbb{R}^{+}$,

$$
\begin{aligned}
& \frac{1}{\mu(m, M, f)} \varphi\left(A \sigma_{f} B\right) \leq \frac{1}{\mu(m, M, f)} \\
& \cdot \varphi\left(A^{1 / 2}\left(f^{A \delta B-m}(M) f^{M-A \delta B}(m)\right)^{1 /(M-m)} A^{1 / 2}\right) \\
& \leq \varphi(A) \sigma_{f} \varphi(B) \leq \varphi(A)^{1 / 2} \\
& \cdot\left(f^{\varphi(A) \delta \varphi(B)-m}(M) f^{M-\varphi(A) \delta \varphi(B)}(m)\right)^{1 /(M-m)} \\
& \cdot \varphi(A)^{1 / 2} \leq \mu(m, M, f) \varphi\left(A \sigma_{f} B\right) .
\end{aligned}
$$

Proof. From the assumption $m A \leq B \leq M A$, we have $m \leq$ $A \delta B:=A^{-1 / 2} B A^{-1 / 2} \leq M$. Therefore, if $f$ is log-convex on $[m, M]$, Proposition 3 implies

$$
\begin{aligned}
& \frac{1}{\mu(m, M, f)} \psi(f(A \delta B)) \leq \frac{1}{\mu(m, M, f)} \\
& \cdot \psi\left(\left(f^{A \delta B-m}(M) f^{M-A \delta B}(m)\right)^{1 /(M-m)}\right) \\
& \leq f(\psi(A \delta B)) \\
& \leq\left(f^{\psi(A \delta B)-m}(M) f^{M-\psi(A \delta B)}(m)\right)^{1 /(M-m)} \\
& \leq \mu(m, M, f) \psi(f(A \delta B)),
\end{aligned}
$$

for any normalized positive linear map $\psi$. In particular, for the given $\varphi$, define

$$
\psi(X)=\varphi(A)^{-1 / 2} \varphi\left(A^{1 / 2} X A^{1 / 2}\right) \varphi(A)^{-1 / 2}
$$

Then, $\psi$ is a normalized linear mapping and the above inequalities imply, upon conjugating with $\varphi(A)^{1 / 2}$, the desired inequalities.

In particular, Corollary 6 can be utilized to obtain versions for the geometric and harmonic operator means, as follows.

Corollary 7. Let $A, B \in \mathscr{B}^{+}(\mathscr{H})$ be such that $m A \leq B \leq$ $M$ A for some positive scalars $m, M$. Then, for any linear map $\varphi$ (not necessarily normalized) and for $t<0$,

$$
\begin{aligned}
\frac{1}{K(m, M, t)} \varphi\left(A \sharp_{t} B\right) & \leq \frac{1}{K(m, M, t)} \varphi\left(A \sigma_{g} B\right) \\
& \leq \varphi(A) \sharp_{t} \varphi(B) \\
& \leq \varphi(A) \sigma_{g} \varphi(B) \\
& \leq K(m, M, t) \varphi\left(A \sharp_{t} B\right),
\end{aligned}
$$

where $g(x)=\left(M^{x-m} m^{M-x}\right)^{t /(M-m)}$ and $K(m, M, t)$ is as in Corollary 4.

Proof. Noting that the function $f(x)=x^{t}$ is log-convex on $[m, M]$ for $t<0$, the result follows by direct application of Corollary 6.

Remark 8. Recently in [7, Theorem 2.2], the authors proved that if $A, B$ are two positive operators, then

$$
\varphi(A) \sharp_{t} \varphi(B) \leq \varphi\left(A \sharp_{t} B\right) \quad t \in[-1,0) .
$$

Therefore, Corollary 7 can be regarded as an extension and a reverse for the above inequality, under the assumption $m A \leq$ $B \leq M A$ with $M \geq m>0$. 
Corollary 9. Let $A, B \in \mathscr{B}^{+}(\mathscr{H})$ be such that $m A \leq B \leq$ $M A$ for some positive scalars $m, M \geq 1$. Then, for any linear map $\varphi$ (not necessarily normalized) and for $t<0$,

$$
\begin{aligned}
\frac{1}{H(m, M, t)} \varphi\left(A !_{t} B\right) & \leq \frac{1}{H(m, M, t)} \varphi\left(A \sigma_{g} B\right) \\
& \leq \varphi(A) !_{t} \varphi(B) \leq \varphi(A) \sigma_{g} \varphi(B) \\
& \leq H(m, M, t) \varphi\left(A !_{t} B\right)
\end{aligned}
$$

where $g(x)=\left(\left(1 !_{t} M\right)^{x-m}\left(1 !_{t} m\right)^{M-x}\right)^{1 /(M-m)}$ and

$$
\begin{aligned}
H & (m, M, t) \\
& =\left[(1-t)^{2}+\frac{t}{m M}(2(1-t) \sqrt{m M}+t)\right]\left(1 !_{t} m\right) \\
& \cdot\left(1 !_{t} M\right) .
\end{aligned}
$$

Proof. Noting that the function $f(x)=\left(1-t+t \quad x^{-1}\right)^{-1}$ is $\log$-convex on $[m, M]$ for $t<0$, provided that $m \geq 1$, the result follows by direct application of Corollary 6 .

We should remark that the mapping $t \longmapsto H(m, M, t)$ is a decreasing function for $t<0$. In particular,

$$
\begin{aligned}
H(m, M, 0) & =1 \leq H(m, M, t) \leq \frac{(\sqrt{m M}-1)^{2}}{(m-1)(M-1)} \\
& =\lim _{t \rightarrow-\infty} H(m, M, t), \quad \forall t<0 .
\end{aligned}
$$

Further, utilizing (8), we obtain the following. In this result and later in the paper, we adopt the notations:

$$
\begin{aligned}
\alpha\left(f, t_{0}\right) & =\frac{a_{f}}{f^{\prime}\left(t_{0}\right)} \\
\text { and } \beta\left(f, t_{0}\right) & =a_{f} t_{0}+b_{f}-\frac{a_{f} f\left(t_{0}\right)}{f^{\prime}\left(t_{0}\right)} .
\end{aligned}
$$

The following proposition gives a simplified special case of [3, Theorem 2.1].

Proposition 10. Let $f:[m, M] \longrightarrow \mathbb{R}^{+}$be convex, $A \in$ $\mathscr{B}_{[m, M]}^{+}(\mathscr{H})$, and $\varphi$ be a normalized positive linear map. If $f$ is either increasing or decreasing on $[m, M]$, then, for any $t_{0} \in(m, M)$,

$$
\varphi(f(A)) \leq \alpha\left(f, t_{0}\right) f(\varphi(A))+\beta\left(f, t_{0}\right),
$$

and

$$
f(\varphi(A)) \leq \alpha\left(f, t_{0}\right) \varphi(f(A))+\beta\left(f, t_{0}\right)
$$

provided that $f^{\prime}\left(t_{0}\right)$ exists and $f^{\prime}\left(t_{0}\right) \neq 0$. Further, both inequalities are reversed if $f$ is concave.
Proof. We give the proof for the reader's convenience. Notice first that $f$ being either increasing or decreasing ensures that $a_{f} f^{\prime}\left(t_{0}\right)>0$. Using a standard functional calculus in (7) with $t=A$ and applying $\varphi$ to both sides imply

$$
\begin{aligned}
& f\left(t_{0}\right)+f^{\prime}\left(t_{0}\right)\left(\varphi(A)-t_{0}\right) \leq \varphi(f(A)) \\
& \quad \leq a_{f} \varphi(A)+b_{f} .
\end{aligned}
$$

On the other hand, applying the functional calculus argument with $t=\varphi(A)$ implies

$$
\begin{aligned}
& f\left(t_{0}\right)+f^{\prime}\left(t_{0}\right)\left(\varphi(A)-t_{0}\right) \leq f(\varphi(A)) \\
& \quad \leq a_{f} \varphi(A)+b_{f} .
\end{aligned}
$$

Noting that $a_{f}$ and $f^{\prime}\left(t_{0}\right)$ have the same sign, both desired inequalities follow from (33) and (34).

Now if $f$ was concave, replacing $f$ with $-f$ and noting linearity of $\varphi$ imply the desired inequalities for a concave function.

As an application, we present the following result, which has been shown in [3, Corollary 2.8].

Corollary 11. Let $A \in \mathscr{B}_{[m, M]}^{+}(\mathscr{H})$. Then, for a normalized positive linear mapping $\varphi$,

$$
\varphi\left(A^{-1}\right) \leq(\varphi(A))^{-1}+\left(\frac{1}{\sqrt{m}}-\frac{1}{\sqrt{M}}\right)^{2}
$$

and

$$
\varphi\left(A^{-1}\right) \leq \frac{(M+m)^{2}}{4 m M}(\varphi(A))^{-1} .
$$

Proof. Let $f(t)=t^{-1}$. Then $f$ is convex and monotone on $[m, M]$. Letting $t_{0}=\sqrt{m M} \in(m, M)$, direct calculations show that $\alpha\left(f, t_{0}\right)=1, \beta\left(f, t_{0}\right)=(1 / \sqrt{m}-1 / \sqrt{M})^{2}$. Then inequality (31) implies the first inequality. The second inequality follows similarly by letting $t_{0}=(m+M) / 2$.

Manipulating Proposition 10 implies several extensions for log-convex functions, as we shall see next.

We will adopt the following constants in Theorem A.

$a_{h}=a[m, M, h], b_{h}=b[m, M, h], \alpha=\alpha\left(h, t_{0}\right), \beta=$ $\beta\left(h, t_{0}\right)$ for $h(t)=\left(f^{t-m}(M) f^{M-t}(m)\right)^{1 /(M-m)}$ and $a_{h_{1}}=$ $a\left[f^{M-m}(m), f^{M-m}(M), h_{1}\right], b_{h_{1}}=b\left[f^{M-m}(m), f^{M-m}(M)\right.$, $\left.h_{1}\right], \alpha_{1}=\alpha\left(h_{1}, t_{1}\right)$, and $\beta_{1}=\beta\left(h_{1}, t_{1}\right)$ for $h_{1}(t)=t^{1 /(M-m)}$.

The first two inequalities of the next result should be compared with Proposition 3, where a reverse-type is presented now.

Theorem A. Let $f:[m, M] \longrightarrow \mathbb{R}^{+}$be log-convex, $A \in$ $\mathscr{B}_{[m, M]}^{+}(\mathscr{H})$, and $\varphi$ be a normalized positive linear map. Then, for any $t_{0}, t_{1} \in(m, M)$, 


$$
\begin{aligned}
f(\varphi(A)) & \leq\left(f^{\varphi(A)-m}(M) f^{M-\varphi(A)}(m)\right)^{1 /(M-m)} \leq \alpha \varphi\left(f^{(A-m) /(M-m)}(M) f^{(M-A) /(M-m)}(m)\right)+\beta \\
& \leq \begin{cases}\alpha\left(\varphi\left(f^{A-m}(M) f^{M-A}(m)\right)\right)^{1 /(M-m)}+\beta, & M-m \geq 1 \\
\alpha \alpha_{1}\left(\varphi\left(f^{A-m}(M) f^{M-A}(m)\right)\right)^{1 /(M-m)}+\alpha \beta_{1}+\beta, & M-m<1 .\end{cases}
\end{aligned}
$$

Proof. For $h(t)=\left(f^{t-m}(M) f^{M-t}(m)\right)^{1 /(M-m)}$, we clearly see that $h$ is convex and monotone on $[m, M]$. Notice that

$$
f(\varphi(A)) \leq\left(f^{\varphi(A)-m}(M) f^{M-\varphi(A)}(m)\right)^{1 /(M-m)}
$$

(by the third inequality of Proposition 3)

$$
=h(\varphi(A)) \leq \alpha\left(h, t_{0}\right) \varphi(h(A))+\beta\left(h, t_{0}\right)
$$

$$
\begin{aligned}
= & \alpha\left(h, t_{0}\right) \varphi\left(f^{(A-m) /(M-m)}(M) f^{(M-A) /(M-m)}(m)\right) \\
& +\beta\left(h, t_{0}\right) .
\end{aligned}
$$

This proves the first two inequalities. Now, for the third inequality, assume that $M-m \geq 1$ and let $h_{1}(t)=t^{1 /(M-m)}$. Then the second inequality can be viewed as

$$
f(\varphi(A)) \leq \alpha \varphi\left(h_{1}\left(f^{A-m}(M) f^{M-A}(m)\right)\right)+\beta .
$$

Since $M-m \geq 1$, it follows that $h_{1}$ is operator concave. Therefore, noting (39) and (1), we have

$$
\begin{aligned}
f(\varphi(A)) & \leq \alpha \varphi\left(h_{1}\left(f^{A-m}(M) f^{M-A}(m)\right)\right)+\beta \\
& \leq \alpha h_{1}\left(\varphi\left(f^{A-m}(M) f^{M-A}(m)\right)\right)+\beta,
\end{aligned}
$$

which is the desired inequality in the case $M-m \geq 1$.

Now, if $M-m<1$, the function $h_{1}$ is convex and monotone. Therefore, taking in account (39) and (31), we obtain

$$
\begin{aligned}
f(\varphi(A)) \leq \alpha \varphi\left(h_{1}\left(f^{A-m}(M) f^{M-A}(m)\right)\right)+\beta \\
\leq \alpha\left(\alpha_{1} h_{1}\left(\varphi\left(f^{A-m}(M) f^{M-A}(m)\right)\right)+\beta_{1}\right)+\beta \\
=\alpha \alpha_{1}\left(\varphi\left(f^{A-m}(M) f^{M-A}(m)\right)\right)^{1 /(M-m)}+\alpha \beta_{1} \\
\quad+\beta
\end{aligned}
$$

which completes the proof.

For the same parameters as Theorem A, we have the following comparison too, in which the first two inequalities have been shown in Theorem A.

Corollary 12. Let $f:[m, M] \longrightarrow \mathbb{R}^{+}$be log-convex, $A \in$ $\mathscr{B}_{[m, M]}^{+}(\mathscr{H})$, and $\varphi$ be a normalized positive linear map. Then

$$
\begin{aligned}
& f(\varphi(A)) \leq\left(f^{\varphi(A)-m}(M) f^{M-\varphi(A)}(m)\right)^{1 /(M-m)} \\
& \quad \leq \alpha \varphi\left(f^{(A-m) /(M-m)}(M) f^{(M-A) /(M-m)}(m)\right)+\beta \\
& \quad \leq \alpha \mu(m, M, f) \varphi(f(A))+\beta .
\end{aligned}
$$

Proof. We prove the last inequality. Letting $\psi(X)=X$ be a normalized positive linear map and noting that $\varphi$ is order preserving, the fourth inequality of Proposition 3 implies

$$
\begin{aligned}
\alpha \varphi( & \left.f^{(A-m) /(M-m)}(M) f^{(M-A) /(M-m)}(m)\right)+\beta \\
= & \alpha \varphi\left(f^{(\psi(A)-m) /(M-m)}(M) f^{(M-\psi(A)) /(M-m)}(m)\right) \\
& +\beta \leq \alpha \mu(m, M, f) \varphi(\psi(f(A)))+\beta \\
= & \alpha \mu(m, M, f) \varphi(f(A))+\beta,
\end{aligned}
$$

which is the desired inequality.

For the next result, the following constants will be used.

$\widehat{a}_{h}=a[m, M, \widehat{h}], \widehat{b}_{h}=b[m, M, \widehat{h}], \widehat{\alpha}=\widehat{\alpha}\left(\widehat{h}, t_{0}\right), \widehat{\beta}=$ $\widehat{\beta}\left(\widehat{h}, t_{0}\right)$ for $\hat{h}(t)=f^{t-m}(M) f^{M-t}(m)$ and $a_{h_{1}}=$ $a\left[f^{M-m}(m), f^{M-m}(M), h_{1}\right], b_{h_{1}}=b\left[f^{M-m}(m), f^{M-m}(M)\right.$, $\left.h_{1}\right], \alpha_{1}=\alpha\left(h_{1}, t_{1}\right)$, and $\beta_{1}=\beta\left(h_{1}, t_{1}\right)$ for $h_{1}(t)=t^{1 /(M-m)}$.

Theorem B. Let $f:[m, M] \longrightarrow \mathbb{R}^{+}$be log-convex, $t_{0}, t_{1} \in$ $(m, M), A \in \mathscr{B}_{[m, M]}^{+}(\mathscr{H})$, and $\varphi$ be a normalized positive linear map. If $M-m \geq 1$,

$$
\begin{aligned}
& \varphi(f(A)) \leq\left[\varphi\left(f^{A-m}(M) f^{M-A}(m)\right)\right] \frac{1}{M-m} \\
& \quad \leq\left(\widehat{\alpha} f^{\varphi(A)-m}(M) f^{M-\varphi(A)}(m)+\widehat{\beta}\right) \frac{1}{M-m} .
\end{aligned}
$$

On the other hand, if $M-m<1$,

$\varphi(f(A))$

$$
\begin{aligned}
\leq & \alpha_{1}\left[\varphi\left(f^{M-A}(m) f^{A-m}(M)\right)\right]^{1 /(M-m)}+\beta_{1} \\
\leq & \alpha_{1}\left[\widehat{\alpha} f^{\varphi(A)-m}(M) f^{M-\varphi(A)}(m)+\widehat{\beta}\right]^{1 /(M-m)} \\
& +\beta_{1} .
\end{aligned}
$$

Proof. Letting $h_{1}(t)=t^{1 /(M-m)}$ and $\widehat{h}(t)=f^{t-m}(M) f^{M-t}(m)$, we have 


$$
\varphi(f(A)) \leq \varphi\left(h_{1}\left(f^{A-m}(M) f^{M-A}(m)\right)\right)
$$

(by the first inequality of Proposition 3)

$$
\leq h_{1}\left(\varphi\left(f^{A-m}(M) f^{M-A}(m)\right)\right)
$$

(since $h_{1}$ is operator concave)

$$
\begin{aligned}
& =\left[\varphi\left(f^{A-m}(M) f^{M-A}(m)\right)\right]^{1 /(M-m)} \\
& =[\varphi(\widehat{h}(A))]^{1 /(M-m)}
\end{aligned}
$$

$$
\begin{aligned}
& \left(\text { where } \widehat{h}(t)=f^{t-m}(M) f^{M-t}(m)\right) \\
\leq & {[\widehat{\alpha} \widehat{h}(\varphi(A))+\beta]^{1 /(M-m)} \quad(\text { by }(31)) } \\
= & \left(\widehat{\alpha} f^{\varphi(A)-m}(M) f^{M-\varphi(A)}(m)+\widehat{\beta}\right)^{1 /(M-m)},
\end{aligned}
$$

which completes the proof for the case $M-m \geq 1$.

Now if $M-m<1$, we have

$$
\varphi(f(A)) \leq \varphi\left(h_{1}\left(f^{A-m}(M) f^{M-A}(m)\right)\right)
$$

(by the first inequality of Proposition 3)

$$
\begin{aligned}
& \leq \alpha_{1} h_{1}\left(\varphi\left(f^{A-m}(M) f^{M-A}(m)\right)\right)+\beta_{1} \quad \text { (by (31)) } \\
& =\alpha_{1}\left[\varphi\left(f^{A-m}(M) f^{M-A}(m)\right)\right]^{1 /(M-m)}+\beta_{1} \\
& =\alpha_{1}[\varphi(\widehat{h}(A))]^{1 /(M-m)}+\beta_{1} \\
& \leq \alpha_{1}[\widehat{\alpha} \hat{h}(\varphi(A))+\widehat{\beta}]^{1 /(M-m)}+\beta_{1} \quad(\text { by }(31)) \\
& =\alpha_{1}\left[\widehat{\alpha} f^{\varphi(A)-m}(M) f^{M-\varphi(A)}(m)+\widehat{\beta}\right]^{1 /(M-m)}+\beta_{1},
\end{aligned}
$$

which completes the proof.

Remark 13. In both Theorems $\mathrm{A}$ and $\mathrm{B}$, the constants $\alpha$ and $\alpha_{1}$ can be selected to be 1 , as follows. Noting that the function $h$ in both theorems is continuous on $[m, M]$ and differentiable on $(m, M)$, the mean value theorem ensures that $a_{h}=h^{\prime}\left(t_{0}\right)$ for some $t_{0} \in(m, M)$. This implies $\alpha=1$, since we use the notation $\alpha \equiv \alpha\left(h, t_{0}\right)=a_{h} / h^{\prime}\left(t_{0}\right)$. A similar argument applies for $h_{1}$. These values of $t_{0}$ can be easily found.

Moreover, one can find $t_{0}$ so that $\beta\left(h, t_{0}\right)=0$, providing a multiplicative version. Since this is a direct application, we leave the tedious computations to the interested reader.

Utilizing Lemma 2, we obtain the following exponential inequality.

Proposition 14. Let $f:[m, M] \longrightarrow \mathbb{R}^{+}$be log-convex, $t_{0}, t_{1} \in$ $(m, M), A \in \mathscr{B}_{[m, M]}^{+}(\mathscr{H})$, and $\varphi$ be a normalized positive linear map. Then

$$
\begin{aligned}
\varphi(f(A)) \geq & \frac{f\left(t_{0}\right)}{\alpha} \exp \left[\frac{f^{\prime}\left(t_{0}\right)}{f\left(t_{0}\right)}\left(\varphi(A)-t_{0}\right)\right] \\
& -\frac{\beta}{\alpha} f\left(t_{0}\right),
\end{aligned}
$$

and

$$
\begin{aligned}
f(\varphi(A)) \geq & \frac{f\left(t_{0}\right)}{\alpha} \varphi\left(\exp \left[\frac{f^{\prime}\left(t_{0}\right)}{f\left(t_{0}\right)}\left(A-t_{0}\right)\right]\right) \\
& -\frac{\beta}{\alpha} f\left(t_{0}\right),
\end{aligned}
$$

where $\alpha=\alpha\left(k, t_{1}\right)$ and $\beta=\beta\left(k, t_{1}\right)$ for $k(t)=\exp \left[\left(f^{\prime}\left(t_{0}\right) /\right.\right.$ $\left.\left.f\left(t_{0}\right)\right)\left(t-t_{0}\right)\right]$.

Proof. By Lemma 2, we have

$$
f(t) \geq f\left(t_{0}\right) \exp \left[\frac{f^{\prime}\left(t_{0}\right)}{f\left(t_{0}\right)}\left(t-t_{0}\right)\right], \quad m \leq t \leq M .
$$

A functional calculus argument applied to this inequality with $t=A$ implies

$$
\begin{aligned}
\varphi(f(A)) \geq & f\left(t_{0}\right) \varphi\left(\exp \left[\frac{f^{\prime}\left(t_{0}\right)}{f\left(t_{0}\right)}\left(A-t_{0}\right)\right]\right) \\
& =f\left(t_{0}\right) \varphi(k(A)) \geq f\left(t_{0}\right) \frac{k(\varphi(A))-\beta}{\alpha} \\
& =\frac{f\left(t_{0}\right)}{\alpha} \exp \left[\frac{f^{\prime}\left(t_{0}\right)}{f\left(t_{0}\right)}\left(\varphi(A)-t_{0}\right)\right] \\
& -\frac{\beta}{\alpha} f\left(t_{0}\right),
\end{aligned}
$$$$
\text { (by (32)) }
$$

which completes the proof of the first inequality. The second inequality follows similarly using (31).

\section{Further Refinements}

The above results are all based on basic inequalities for convex functions. Therefore, refinements of convex functions inequalities can be used to obtain sharper bounds. We give here some examples. In [11], the following simple inequality was shown for the convex function $f:[m, M] \longrightarrow \mathbb{R}$,

$$
\begin{gathered}
f(t)+\frac{2 \min \{t-m, M-t\}}{M-m}\left(\frac{f(m)+f(M)}{2}\right. \\
\left.-f\left(\frac{m+M}{2}\right)\right) \leq L[m, M, f](t) .
\end{gathered}
$$

This inequality can be used to obtain refinements of (31) and (32) as follows. First, we note that the function $t \longmapsto t_{\min }:=$ $(2 \min \{t-m, M-t\} /(M-m))((f(m)+f(M)) / 2-f((m+$ $M) / 2)$ ) is a continuous function. Further, noting that

$$
\min \{t-m, M-t\}=\frac{M-m+|M+m-2 t|}{2},
$$

one can apply a functional calculus argument on (52). With this convention, we will use the notation

$$
\begin{aligned}
A_{\text {min }}:= & \frac{1}{M-m}\left(\frac{f(m)+f(M)}{2}-f\left(\frac{m+M}{2}\right)\right) \\
& \cdot(M-m+|M+m-A|) .
\end{aligned}
$$


The following is a refinement of Proposition 10. Since the proof is similar to that of Proposition 10 utilizing (52), we do not include it here.

Proposition 15. Let $f:[m, M] \longrightarrow \mathbb{R}^{+}$be convex, $A \in$ $\mathscr{B}_{[m, M]}^{+}(\mathscr{H})$, and $\varphi$ be a normalized positive linear map. If $f$ is either increasing or decreasing on $[m, M]$, then, for any $t_{0} \in(m, M)$,

$$
\begin{gathered}
\varphi(f(A))+\varphi\left(A_{\min }\right) \leq \alpha\left(f, t_{0}\right) f(\varphi(A)) \\
+\beta\left(f, t_{0}\right),
\end{gathered}
$$

and

$$
\begin{aligned}
& f(\varphi(A))+(\varphi(A))_{\min } \\
& \quad \leq \alpha\left(f, t_{0}\right) \varphi(f(A))+\beta\left(f, t_{0}\right),
\end{aligned}
$$

provided that $f^{\prime}\left(t_{0}\right)$ exists and $f^{\prime}\left(t_{0}\right) \neq 0$.

Notice that applying this refinement to the convex function $f(t)=t^{-1}$ implies refinements of both inequalities in Corollary 11 as follows.

Corollary 16. Under the assumptions of Corollary 11, we have

$$
\varphi\left(A^{-1}\right)+\varphi\left(A_{\min }\right) \leq(\varphi(A))^{-1}+\left(\frac{1}{\sqrt{m}}-\frac{1}{\sqrt{M}}\right)^{2}
$$

and

$$
\varphi\left(A^{-1}\right)+(\varphi(A))_{\min } \leq \frac{(M+m)^{2}}{4 m M}(\varphi(A))^{-1} .
$$

Remark 17. Inequality (52) has been studied extensively in the literature, where numerous refining terms have been found. We refer the reader to $[12,13]$, where a comprehensive discussion has been made therein. These refinements then can be used to obtain further refining terms for Proposition 10 .

Further, these refinements can be applied to log-convex functions too. This refining approach leads to refinements of most inequalities presented in this article; where convexity was the key idea. We leave the detailed computations to the interested reader.

\section{Data Availability}

All data generated or analysed during this study are included in this published article. There is no experimental data in this article.

\section{Conflicts of Interest}

The authors declare that they have no conflicts of interest.

\section{Acknowledgments}

The work of the first author (Mohammad Sababheh) is supported by a sabbatical leave from Princess Sumaya University for technology. Shigeru Furuichi was partially supported by JSPS KAKENHI Grant Number 16K05257.

\section{References}

[1] M. D. Choi, "A Schwarz inequality for positive linear maps on $C^{*}$-algebras," Illinois Journal of Mathematics, vol. 18, pp. 565574,1974

[2] C. Davis, "A Schwarz inequality for convex operator functions," Proceedings of the American Mathematical Society, vol. 8, pp. 4244, 1957.

[3] J. Mićić, J. Pecarić, and Y. Seo, "Complementary inequalities to inequalities of Jensen and Ando based on the Mond-Pecarić Method," Linear Algebra and Its Applications, vol. 318, no. 1-3, pp. 87-107, 2000.

[4] C.-K. Li and R. Mathias, "Matrix inequalities involving a positive linear map," Linear and Multilinear Algebra, vol. 41, no. 3, pp. 221-231, 1996.

[5] H. R. Moradi, I. H. Gümüş, and Z. Heydarbeygi, "A glimpse at the operator Kantorovich inequality," Linear and Multilinear Algebra, pp. 1-6, 2018.

[6] B. Mond and J. E. Pecarić, "Operator convex functions of several variables," Soochow Journal of Mathematics, vol. 24, no. 4, pp. 239-254, 1998.

[7] J. I. Fujii and Y. Seo, "Tsallis relative operator entropy with negative parameters," Advances in Operator Theory, vol. 1, no. 2, pp. 219-235, 2016.

[8] J. Mićić, J. Pecarić, Y. Seo, and M. Tominaga, "Inequalities for positive linear maps on Hermitian matrices," Mathematical Inequalities \& Applications, vol. 3, no. 4, pp. 559-591, 2000.

[9] S. S. Dragomir, "Some Jensen's type inequalities for log-convex functions of selfadjoint operators in Hilbert spaces," Bulletin of the Malaysian Mathematical Sciences Society, vol. 34, no. 3, pp. 445-454, 2011.

[10] J. Mićić and J. Pecarić, "Order among power means of positive operators, II," Scientiae Mathematicae Japonicae, vol. 71, no. 1, pp. 93-109, 2010.

[11] F.-C. Mitroi, "About the precision in Jensen-Steffensen inequality, Annals of the University of Craiova, vol. 37, no. 4, pp. 73-84, 2010.

[12] M. Sababheh, "Improved Jensen's inequality," Mathematical Inequalities \& Applications, vol. 20, no. 2, pp. 389-403, 2017.

[13] M. Sababheh, "Means refinements via convexity," Mediterranean Journal of Mathematics, vol. 14, no. 3, 2017. 


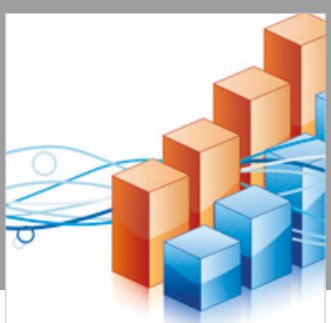

Advances in

Operations Research

\section{-n-m}
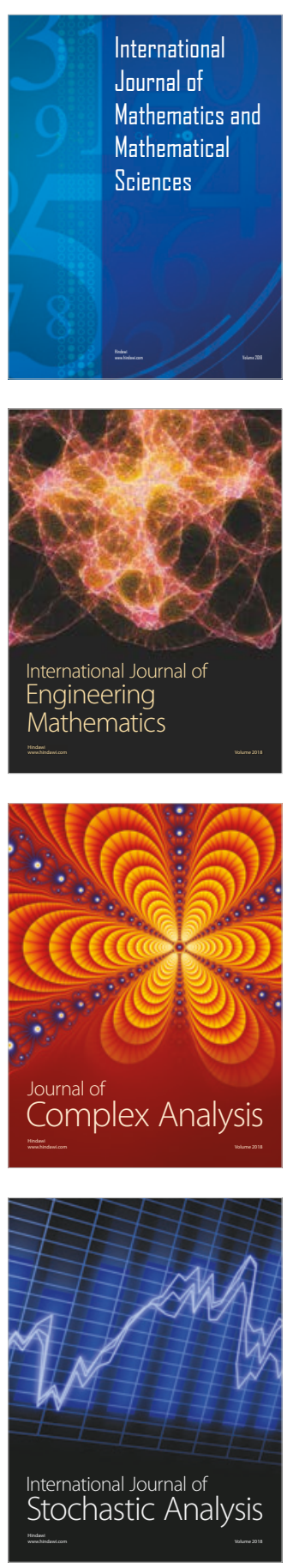
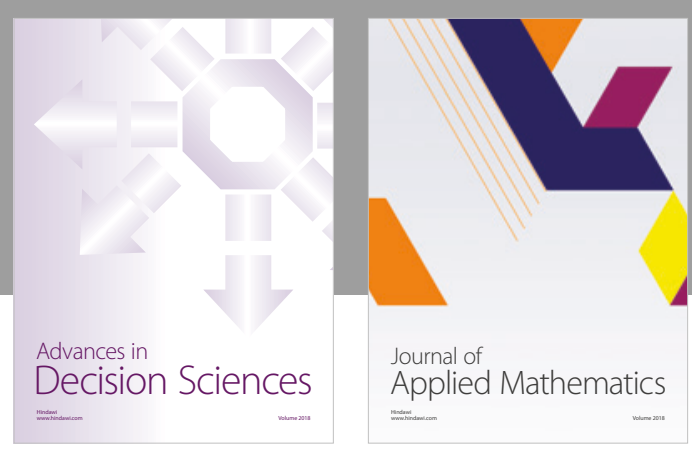

Journal of

Applied Mathematics
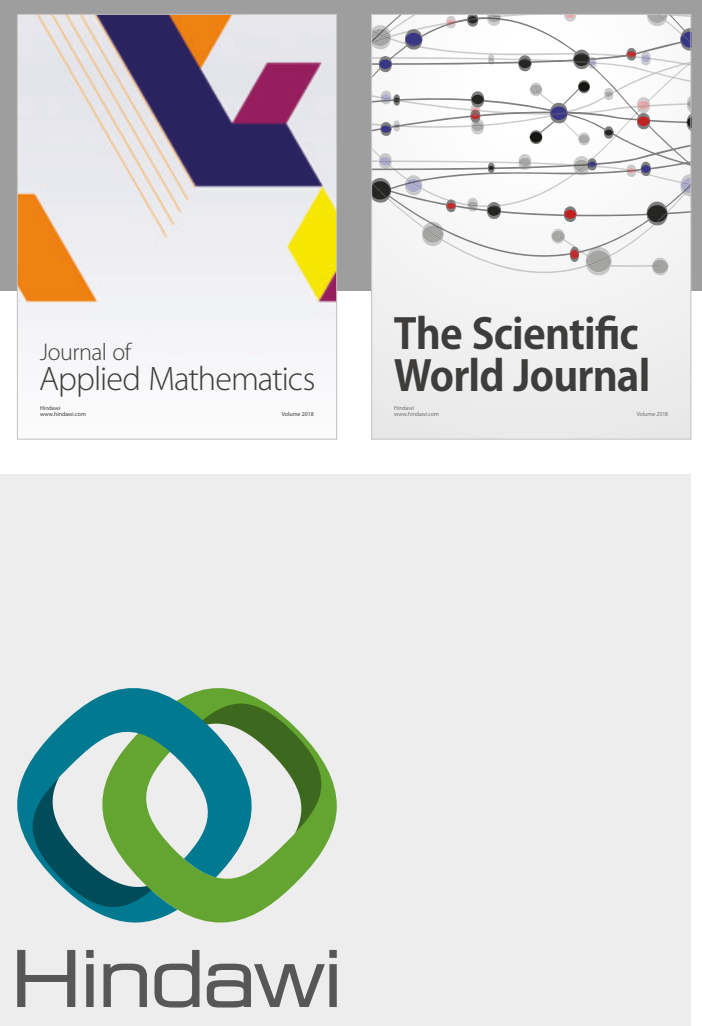

Submit your manuscripts at

www.hindawi.com

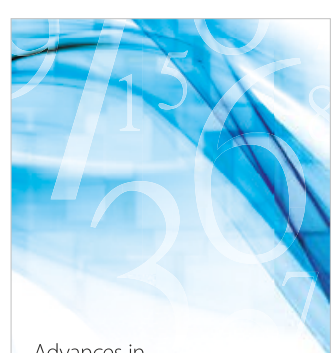

Advances in
Numerical Analysis
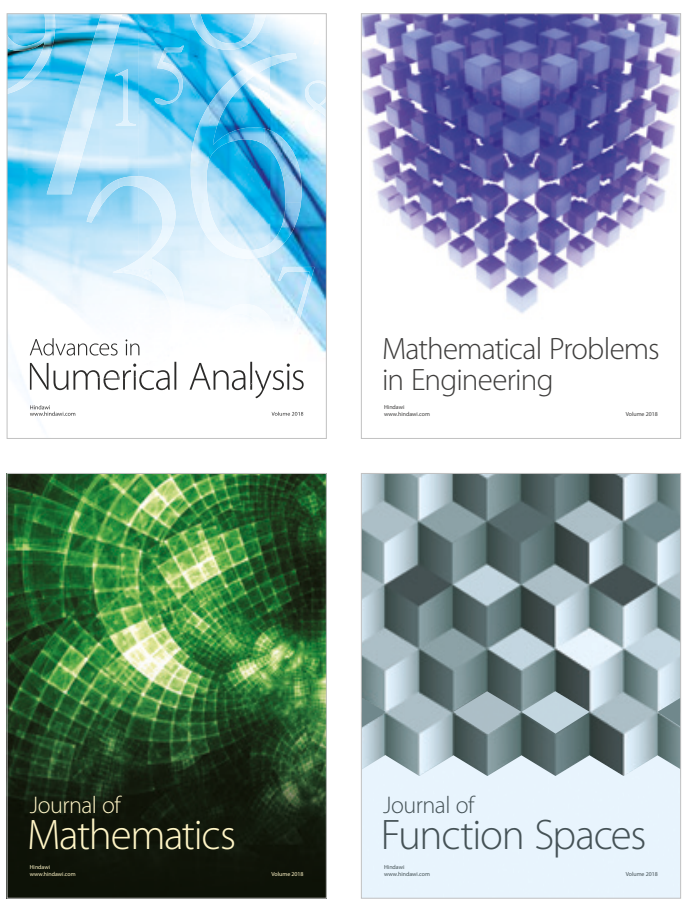

Mathematical Problems in Engineering

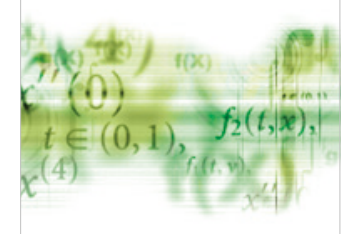

International Journal of

Differential Equations

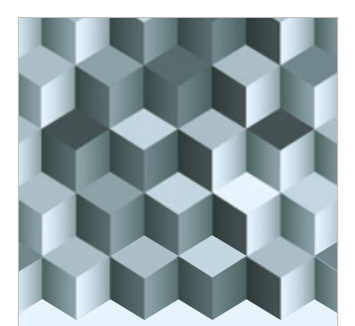

Journal of

Function Spaces

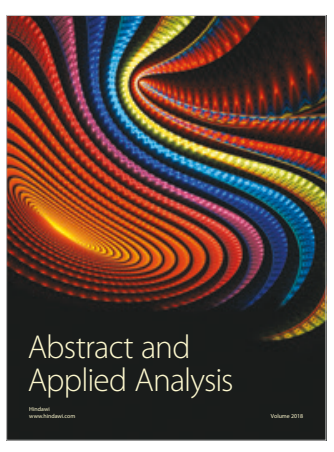

The Scientific

World Journal

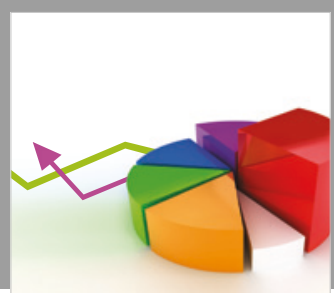

Journal of

Probability and Statistics
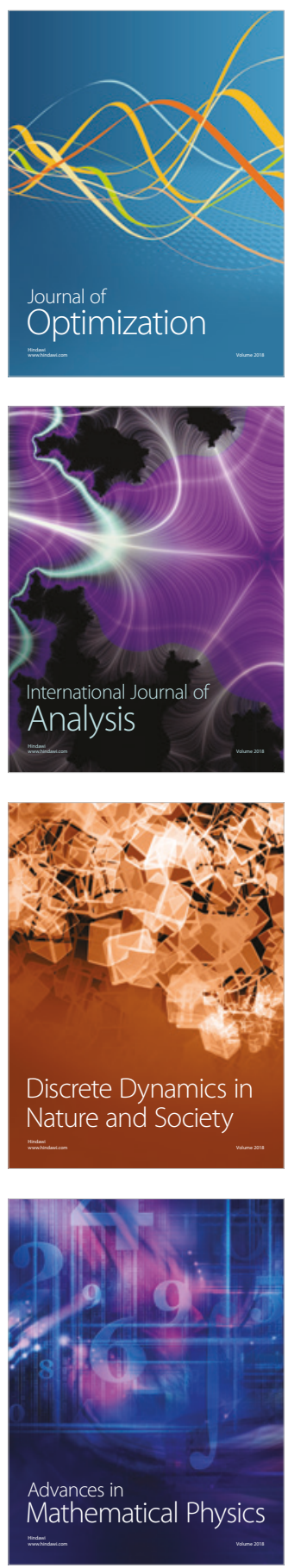